\title{
A modular Raman microspectroscopy system for biological tissue analysis
}

\author{
Shuang Wang ${ }^{\mathrm{a}, \mathrm{b}, \mathrm{c}}$, Jianhua Zhao ${ }^{\mathrm{a}, \mathrm{b}}$, Harvey Lui ${ }^{\mathrm{a}, \mathrm{b}}$, Qingli $\mathrm{He}^{\mathrm{c}}$ and Haishan Zeng ${ }^{\mathrm{a}, \mathrm{b}, *}$ \\ ${ }^{a}$ Cancer Imaging Department, British Columbia Cancer Agency Research Centre, Vancouver, BC, \\ Canada \\ ${ }^{\mathrm{b}}$ Laboratory for Advanced Medical Photonics, Photomedicine Institute, Department of Dermatology \\ and Skin Science, University of British Columbia and Vancouver Coastal Health Research Institute, \\ Vancouver, BC, Canada \\ ${ }^{\mathrm{c}}$ Department of Physics, Northwest University, Xi'an, Shaanxi, China
}

\begin{abstract}
Raman spectroscopy has been used as a sensitive tool for studying biological tissue and evaluating disease. In many applications, microscopic level resolution spectral analysis is desirable. And this has been performed mostly by expensive commercial confocal micro-Raman systems. In this research, we present a simple method for building an economical and modular Raman microspectroscopy system that combines a microscope with a Raman spectrometer using an optical fiber bundle. The bundle with a circular collection end is positioned at an image plane of the microscope to collect Raman signals from the interested micro-location on the sample. The light delivery end is specially configured so that its 37 fibers are arranged along a straight line to fit into the spectrometer entrance slit. This configuration improves light collection efficiency and maintains high spectral resolution. To battle the great background autofluorescence and Raman signals that could originate from the microscope slides and optics due to the non-confocal set-up of our simplified system, conventional normal-incident illumination is replaced by oblique illumination at $45^{\circ}$ degrees and the microscope slides are coated with gold. We demonstrated the usefulness of the system by measuring micro-Raman spectra from different skin layers on vertical sections of normal skin tissue samples.
\end{abstract}

Keywords: Microscopy Raman, near infrared fluorescence imaging, gold-coated slide, oblique illumination, tissue Raman spectroscopy, skin

\section{Introduction}

Raman microspectroscopy has been well established as a sensitive method for studying cells, tissues and diseases [8]. Although the native Raman signal from biological materials is somewhat weak due to the low probability of inelastic scattering events, those Raman scattered signals that can be captured and recorded provide specific molecular information about the biochemical composition and structure of cells and tissues [3,9]. Conventional Raman spectroscopy consists of a light source, typically a laser, and systems for light delivery, collection and signal detection. Recent technical advances in confocal microRaman spectroscopy provide more precise spatial interrogation and become the preferred analysis in many applications $[3,9]$. However, this has been performed mostly by expensive commercial confocal micro-Raman systems. Having less expensive and simpler systems available in most laboratories will further expand the applications of micro-Raman analysis.

\footnotetext{
${ }^{*}$ Corresponding author: Dr. Haishan Zeng, Cancer Imaging Department, British Columbia Cancer Agency Research Centre, 675 West 10th Avenue, Vancouver, BC, V5Z 1L3, Canada. Tel.: +1 604675 8083; Fax: +1 604675 8099; E-mail: hzeng@ bccrc.ca.
} 
A major advantage of Raman microspectroscopy for study of biological samples is the minimal sample preparation required [7]. Cells and tissues are usually grown or placed on an appropriate IR-transparent substrate such as $\mathrm{CaF}_{2}, \mathrm{BaF}_{2}, \mathrm{MgF}_{2}$ and $\mathrm{ZnSe}[7,8]$. However, these particular materials exhibit autofluorescence and/or Raman scattering, which can contaminate the desired measurement in the form of background signals especially for non-confocal configurations. These substrates are also expensive and have undesirable mechanical properties such as fragility.

We introduce an economical and modular dispersive Raman microspectroscopy system with low background signal by combining a Raman spectrometer with a microscope through fiber optics and employing gold-coated microscope slides. By using glass slides coated with a layer of gold for mounting thin tissue sections and oblique illumination, we are able to avoid most of the unwanted background signal from the system and the microscope slide. To enhance the collection efficiency of the inherently weak Raman signals, we packed thirty-seven $40 \mu \mathrm{m}$ core-diameter fibers into a bundle. Sample Raman spectra from normal skin sections using this microspectroscopy system are presented.

\section{Materials and methods}

Raman microspectroscopy system. The Raman microspectroscopy system was developed by modifying a microspectrophotometer system originally constructed for fluorescence measurements [10]. The setup of the Raman microspectroscopy system is schematically shown in Fig. 1. Its major components include light sources (a laser and a tungsten white light lamp), an inverted microscope (Diaphot, Nikon) and a Raman spectrometer.

For measuring Raman spectra, $785 \mathrm{~nm}$ near infrared (NIR) light from a fiber-coupled, temperature stabilized diode laser (BRW-785-1.0-100-0.22-SMA, B\&W Tek Inc., Newark, DE, USA) was delivered to a collimator, passing through a band-pass filter (FF01-785/62-25, Semrock Inc., Rochester, NY, USA) and then focused by a biconvex lens onto the tissue section at an angle of $45^{\circ}$. The Raman signal was then collected by the subjacent objective, passed through a $830 \mathrm{~nm}$ longpass filter (LP02-830RU-25, Semrock, Rochester, NY, USA) and focused onto the image plane of the camera port of the microscope.

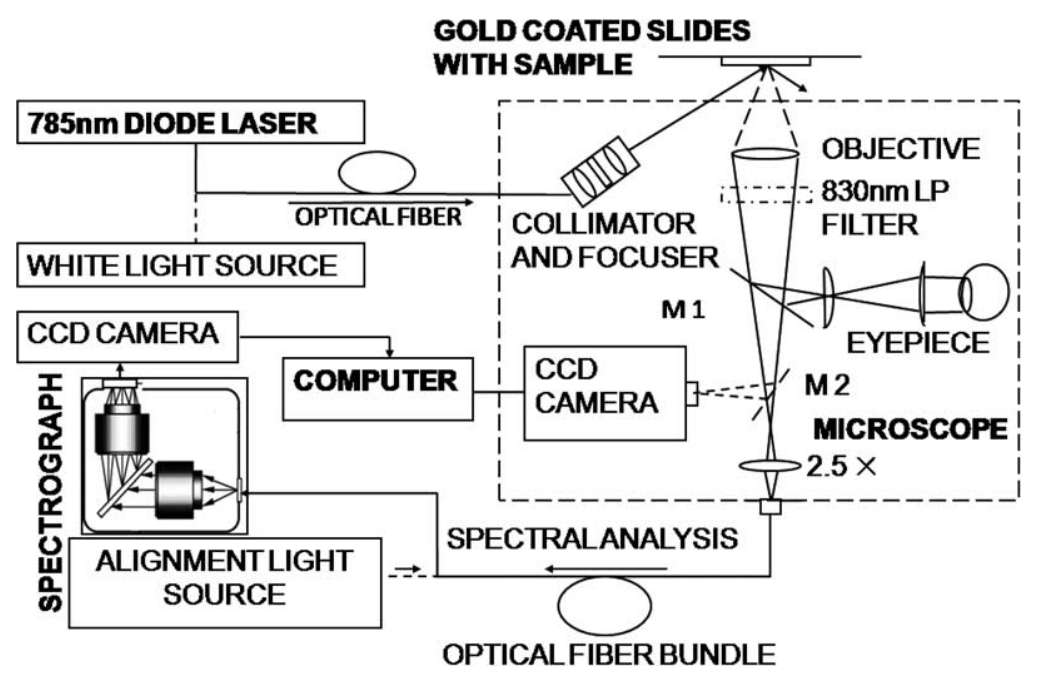

Fig. 1. The setup of the Raman microspectroscopy system. 
A customized optical fiber bundle (FiberTech Optica, ON, Canada) consisting of thirty-seven $40 \mu \mathrm{m}$ optical fibers was used to enhance the collection efficiency of the inherently weak Raman signals. At the collection end connecting to the microscope, the fibers were packed into a $0.385 \mathrm{~mm}$ diameter circular area, which also defined the detection spot on the sample, while in the light delivery end connecting to the spectrometer, they were packed into two paralleled columns to fit into the entrance slit of the spectrograph. One column contains 18 and the other contains 19 of the $40 \mu \mathrm{m}$ core-diameter optical fibers. The spectrometer is equipped with an NIR-optimized back-illuminated deep-depletion CCD array (Spec-10:100BR/LN, Princeton Instruments, Trenton, NJ, USA) and a transmissive imaging spectrograph (HoloSpec-f/2.2-NIR, Kaiser Optical, Ann Arbor, MI, USA) with a holographic grating (HSG-785-LF, Kaiser Optical, Ann Arbor, MI, USA). The CCD has a 16 bit dynamic range and is liquid nitrogen cooled to $-120^{\circ} \mathrm{C}$. The spectral resolution of the system is $8 \mathrm{~cm}^{-1}$. The spectrometer is controlled by a computer (Optiplex 755, Dell, TX, USA) through a USB 2.0 interface. The wavelength was calibrated using an Hg-Ar lamp (HG-1, Ocean Optics Inc., Dunedin, FL, USA) and the spectral response of the system was calibrated using an NIST traceable tungsten calibration lamp (RS-3, EG\&G Gamma Scientific, San Diego, CA, USA) [11]. The data acquisition was implemented with WinSpec software package (drivers from Roper Scientific, Trenton, NY, USA). The dark noise of the CCD detector was measured and removed from each acquired spectrum. The integration time for each spectrum is $30 \mathrm{~s}$.

White light illumination was used for conventional microscopic imaging, which can be seen through the eyepiece. To locate the spot for Raman spectra measurement, the collection optical fiber bundle was disconnected from the spectrometer and connected to an auxiliary white light source. The light from the fiber tips was focused on the sample, which was visible through the eyepiece as the image of the fiber bundle end face. Based on the property of light reversibility, only the signal from this bright spot could be collected by the fiber bundle for spectral analysis. This bright spot was therefore used to localize the desired spot (micro-location) for spectral measurement [10]. For the measurements shown in this paper, the spot size was about $15.4 \mu \mathrm{m}$ in diameter for a $10 \times$ objective and a $0.385 \mathrm{~mm}$ diameter collection fiber bundle.

The system also provides for near-infrared fluorescence imaging of the sample. If the movable mirror M2 is inserted into the light path in the microscope system (Fig. 1), the fluorescence signal (including the associated weak Raman signal) from the sample will be deflected and form an image on an NIRenhanced CCD camera (Alta ${ }^{\circledR}$ U1, Apogee Instruments Inc., Roseville, CA, USA) through the video port on the microscope. Fluorescence images were acquired by the computer for storage and/or further processing. Also, the visible image of the sample could be acquired with a visible light source in the same way.

Microscope slide background fluorescence. For micro-Raman measurements and NIR fluorescence imaging, it is critical to choose a substrate (slide) material that is relatively free of intrinsic fluorescence and Raman scattering because these background signals from the slide may contaminate the Raman spectra and the fluorescence images. We measured a standard glass slide (Cat. No. 12-544-1, Fisher Scientific, Pittsburgh, PA, USA), a cover slip (Cat. No. 12-545E, Fisher Scientific, Pittsburgh, PA, USA) and $\mathrm{BaF}_{2}$ slide. All presented significant fluorescence and Raman background as shown in Fig. 2. To reduce the background fluorescence and Raman signals, the glass slide coated with a layer of $120 \mathrm{~nm}$ thick pure gold (Biogold ${ }^{\circledR}$ 63479-AS, Electron Microscopy Sciences, Hatfield, PA, USA), was utilized in our system and shows near zero background fluorescence and Raman emitted. In addition to the fact that gold exhibits no NIR fluorescence, the other advantage of this special preparation is that the majority of excitation light is specularly reflected away from the optical axis by the surface gold layer and is therefore not collected by the objective (Fig. 1). This helps to reduce the background fluorescence and 


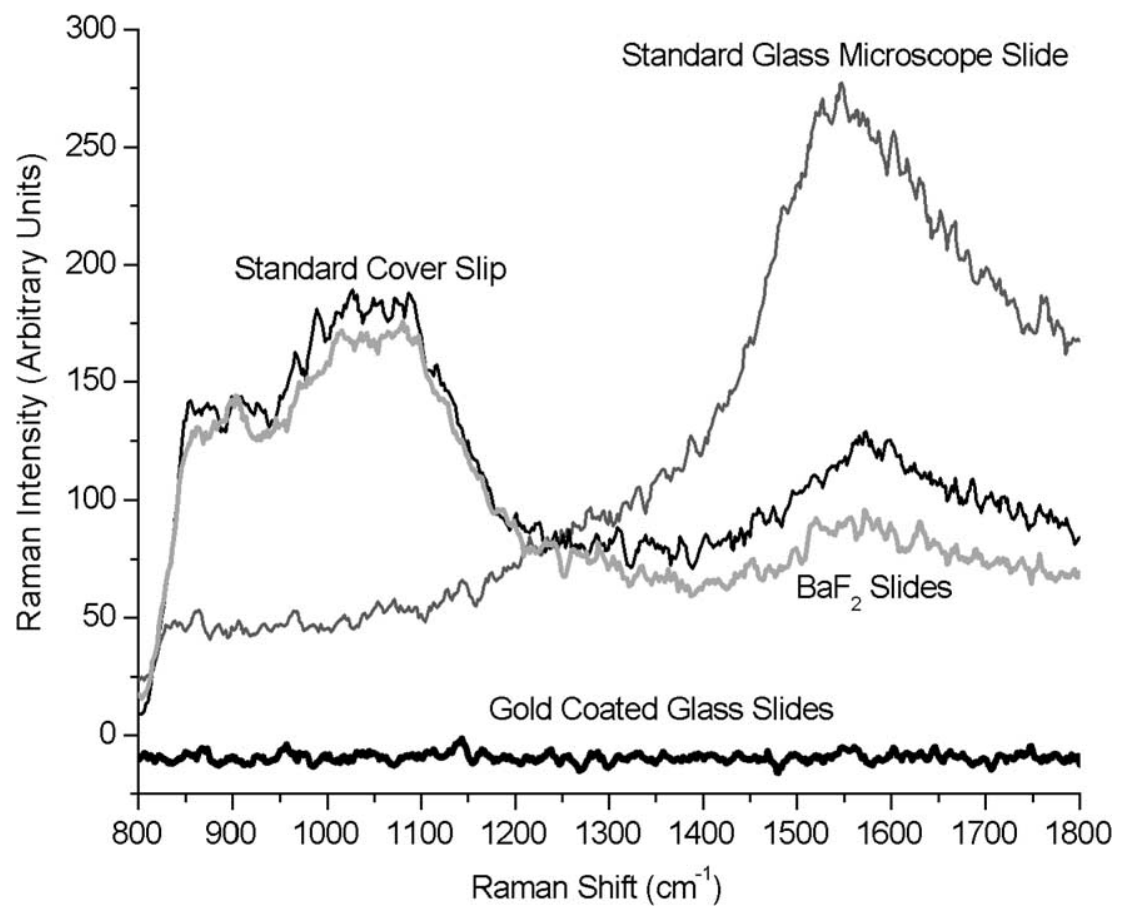

Fig. 2. Fluorescence and Raman background spectra of standard glass microscope slide, standard microscope cover slip, $\mathrm{BaF}_{2}$ slide and gold-coated glass slide.

Raman signals from the objective and other optical components of the microscope that could otherwise be excited by the laser light. Furthermore, when the excitation light is focused onto the tissue, part of it is absorbed and scattered by the skin's molecules and the rest is transmitted through the thin tissue sample. Since most of the transmitted laser beam is subsequently reflected back by the gold layer on the slide, the absorption and scattering occur twice for this arrangement. This optical tissue path doubling scheme increases the inelastic scattering events arising within the skin sample.

Sample preparation. Unstained and fresh normal skin sections for Raman microspectroscopy measurement and NIR autofluorescence imaging were obtained from facial surgery. Immediately after excision, the sample was put into a capsule and snaps frozen with liquid nitrogen. Vertical sections of $20 \mu \mathrm{m}$ thicknesses were cut with a microtome at $-20^{\circ} \mathrm{C}$ and mounted onto gold-coated glass slides without cover slips. The frozen sections were kept in $-80^{\circ} \mathrm{C}$ until usage. Before the measurements, the samples were acclimated to room temperature.

\section{Results and discussion}

The pure Raman spectra of the skin samples were obtained after fluorescence background subtraction from the measured raw spectra using the Vancouver Raman Algorithm we developed previously [11]. Figure 3 shows Raman spectra of different skin layers along with the autofluorescence image of the normal skin sample. This image was used to identify various skin structures. Prominent spectral features in the range of $800-1800 \mathrm{~cm}^{-1}$ are the major vibration bands around 1650, 1445, 1311, 1269, 1080, 1002 and $855 \mathrm{~cm}^{-1}$, which can all be found from the in vivo skin Raman spectra [11]. The strong 


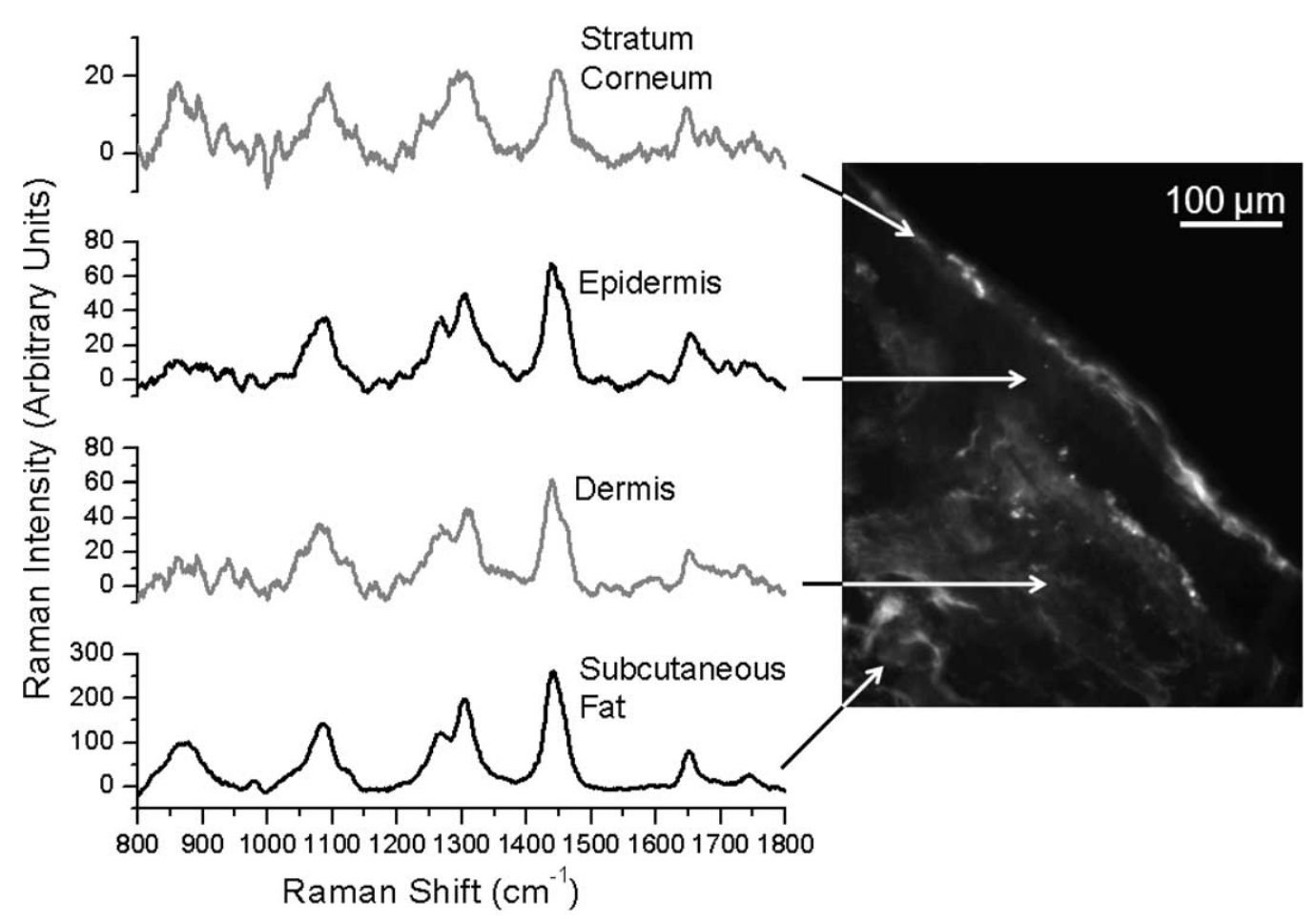

Fig. 3. NIR autofluorescence image of normal facial skin tissue section and Raman spectra measured from different skin layers on the section. Several spectral differences among the major layers of skin (stratum corneum, epidermis, dermis and subcutaneous fat) are apparent due to intrinsic differences in skin layer composition. Integration time is $30 \mathrm{~s}$ for each spectrum.

Raman bands at 1650 and $1269 \mathrm{~cm}^{-1}$ are assigned to protein vibration modes involving amide bonds (amide I and amide III), and mainly originate from keratin which is the most abundant protein in stratum corneum [2]. The next strongest band is at $1445 \mathrm{~cm}^{-1}$, assigned to the $\mathrm{CH}_{2}$ deformation of proteins and lipids. Another strong band centered at $1301 \mathrm{~cm}^{-1}$ was assigned to a twisting deformation of the $\mathrm{CH}_{2}$ methylene groups of intracellular lipid acyl moieties. The region from 1000 to $1150 \mathrm{~cm}^{-1}$ in the Raman spectra contains information on the hydrocarbon chain, while the peak at $1080 \mathrm{~cm}^{-1}$ could be due to a random conformation vibration mode. Also, bands around $855 \mathrm{~cm}^{-1}$ and $945 \mathrm{~cm}^{-1}$ could be observed presumably from collagen. The Raman spectra of normal skin sections obtained in this study are essentially similar to those reported in the literature [1,4,5].

As shown in Fig. 3, the Raman spectra obtained from vertical skin sections reveal significant difference between skin layers. The Raman from the stratum corneum is weak because it is a thin layer. Nevertheless most of them were in agreement with results obtained from FT-Raman measurements on isolated stratum corneum [1,5]. The major variations in the spectra were seen in the relative band intensities, notably around $850-1200 \mathrm{~cm}^{-1}$ and $1250-1350 \mathrm{~cm}^{-1}$. One of the most striking differences between the spectra of the stratum corneum and the underlying epidermis was observed at 1062, 1126 and $1296 \mathrm{~cm}^{-1}$. All these three bands were strong features in the Raman spectrum of ceramide, which represents the most abundant class of stratum corneum lipids [4]. The prominent vibrational features of human skin, such as the $\nu(\mathrm{C}=\mathrm{O})$ amide I band at $1650 \mathrm{~cm}^{-1}$, the $\delta\left(\mathrm{CH}_{3}\right)$ and $\delta\left(\mathrm{CH}_{2}\right)$ scissoring mode at $1445 \mathrm{~cm}^{-1}$, the $\mathrm{CH}_{2}$ deformation at $1301 \mathrm{~cm}^{-1}$ and the $\nu(\mathrm{CN})$ and $\delta(\mathrm{NH})$ amide II bands at $1269 \mathrm{~cm}^{-1}$, can be clearly discerned in different skin layers. It clearly illustrates that skin Raman properties can be 
easily studied using tissue sections with low background signals by our Raman microspectroscopy system. The results obtained will help explain the biophysical basis for the in vivo skin spectra from clinical measurements, which is an integral contribution of different skin layers.

To the best of our knowledge, this is the first report of microscopic NIR autofluorescence images of the skin. It clearly outlined the NIR fluorophore distributions in the skin. The image shown in Fig. 3 suggests that there are considerable NIR autofluorescence emission from the stratum corneum and the dermis, while the epidermis layer exhibits minimum signals. We are planning to study the microscopic NIR autofluorescence properties of various skin lesions. The results obtained will be used to understand the microscopic origins and biophysical basis of many interesting in vivo NIR autofluorescence images we obtained recently in our clinical studies [6].

\section{Conclusions}

We have successfully built an economical and modular Raman microspectroscopy system that combines a microscope with a Raman spectrometer using an optical fiber bundle. The system has three unique features: (1) a round-to-linear fiber bundle configuration is used to improve Raman signal collection efficiency, while also maintain high spectral resolution; (2) the use of oblique illumination and (3) the use of gold-coated microscope slide effectively eliminated the background autofluorescence and Raman signals that could originate from the microscope slides and optics due to the non-confocal setup of our simplified system. We demonstrated the usefulness of the system by successfully measuring micro-Raman spectra from different skin layers on vertical sections of normal skin tissue samples. Using the system we also captured microscopic near infrared autofluorescence images of the skin for the first time. These microscopic spectral and imaging data will be very helpful for understanding the microscopic origins and biophysical bases of our in vivo clinical Raman spectra and NIR autofluorescence images.

\section{Acknowledgements}

This work was supported by the Canadian Cancer Society, the Canadian Dermatology Foundation, the VGH \& UBC Hospital Foundation "In It for Life Fund" and the BC Hydro Employees Community Services Fund.

\section{References}

[1] A.N.C. Anigbogu, A.C. Williams, B.W. Barry and H.G.M. Edwards, Fourier transform Raman spectroscopy of interactions between the penetration enhancer dimethyl sulfoxide and human stratum corneum, International Journal of Pharmaceutics 125 (1995), 265-282.

[2] B.W. Barry, H.G.M. Edwards and A.C. Wiliams, Fourier transform Raman and infrared vibrational study of human skin: Assignment of spectral bands, Journal of Raman Spectroscopy 23 (1992), 641-645.

[3] P.J. Caspers, G.W. Lucassen, E.A. Carter, H.A. Bruining and G.J. Puppels, In vivo confocal Raman microspectroscopy of the skin: noninvasive determination of molecular concentration profiles, Journal of Investigative Dermatology 116 (2001), 434-442.

[4] P.J. Caspers, G.W. Lucassen, R. Wolthuis, H.A. Bruining and G.J. Puppels, In vitro and in vivo Raman spectroscopy of human skin, Biospectroscopy 4 (1998), S31-S39.

[5] H.G.M. Edwards, A.C. Williams and B.W. Barry, Potential applications of FT-Raman spectroscopy for dermatological diagnostics, Journal of Molecular Structure 347 (1995), 379-387. 
[6] X. Han, H. Lui, D.I. McLean and H. Zeng, Near-infrared autofluorescence imaging of cutaneous melanins and human skin in vivo, Journal of Biomedical Optics 14 (2009), 0240179.

[7] C. Krafft and V. Sergo, Biomedical applications of Raman and infrared spectroscopy to diagnose tissues, Spectroscopy 20 (2006), 195-218.

[8] I. Notingher and L.L. Hench, Raman microspectroscopy: a noninvasive tool for studies of individual living cells in vitro, Expert Review of Medical Devices 3 (2006), 215-234.

[9] M.A. Short, H. Lui, D. McLean, H. Zeng, A. Alajlan and X.K. Chen, Changes in nuclei and peritumoral collagen within nodular basal cell carcinomas via confocal micro-Raman spectroscopy, Journal of Biomedical Optics 11 (2006), 0340041-034004-9.

[10] H. Zeng, C.E. MacAulay and D.I. Mclean, Novel microspectrophotometer and its biomedical applications, Optical Engineering 32 (1993), 1809-1814.

[11] J. Zhao, H. Lui, D.I. McLean and H. Zeng, Integrated real-time Raman system for clinical in vivo skin analysis, Skin Research and Technology 14 (2008), 484-492. 


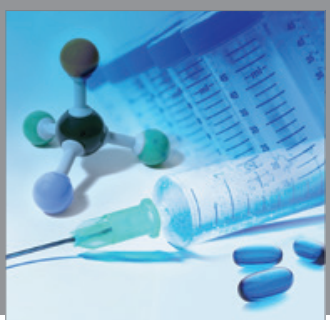

International Journal of

Medicinal Chemistry

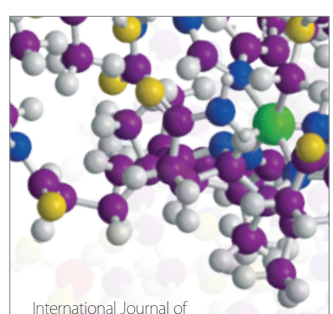

Carbohydrate Chemistry

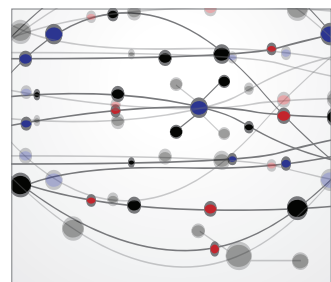

The Scientific World Journal
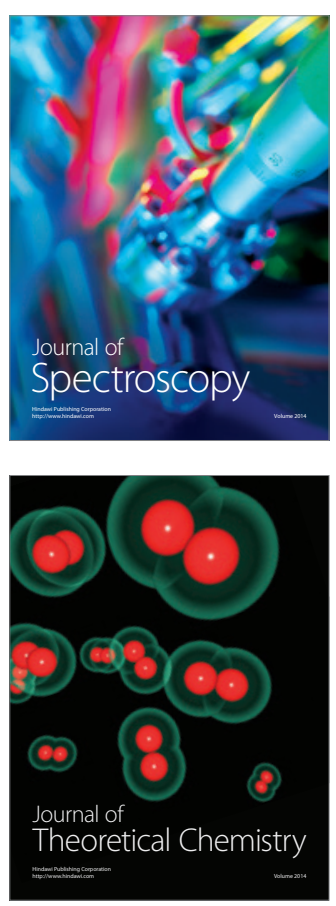
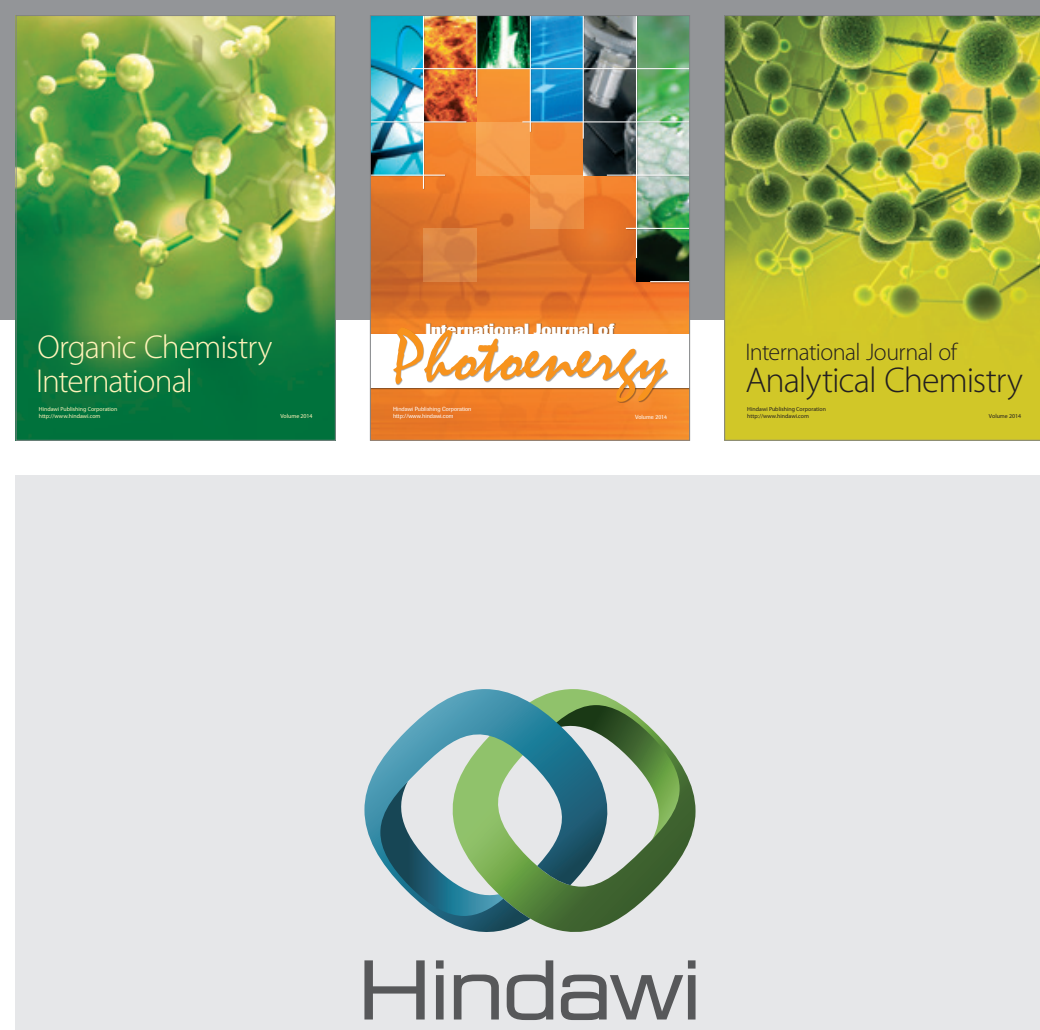

Submit your manuscripts at

http://www.hindawi.com
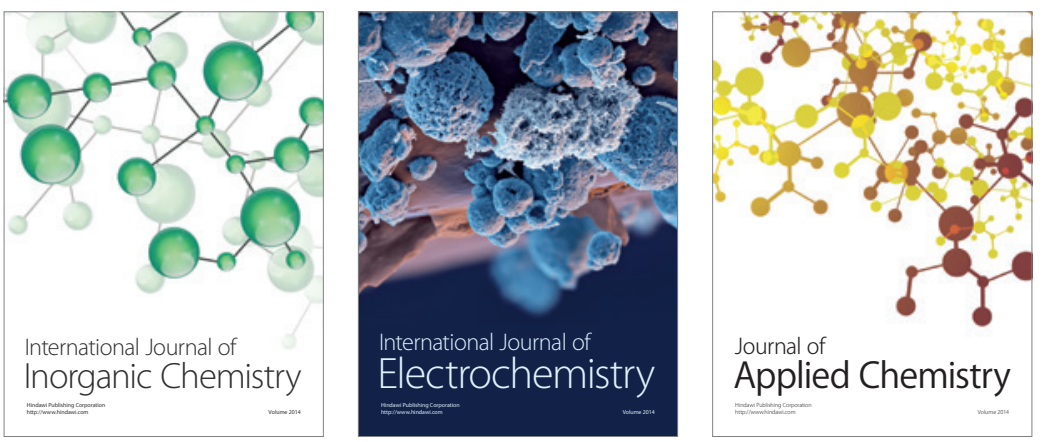

Journal of

Applied Chemistry
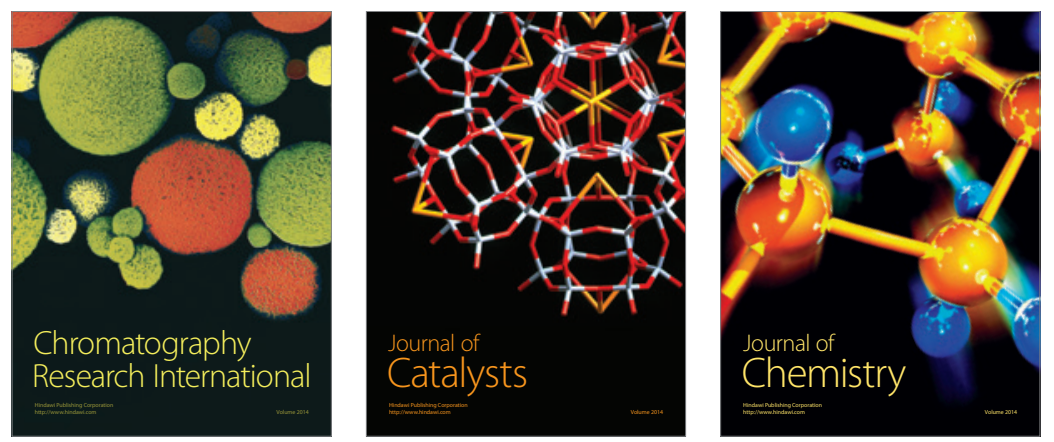
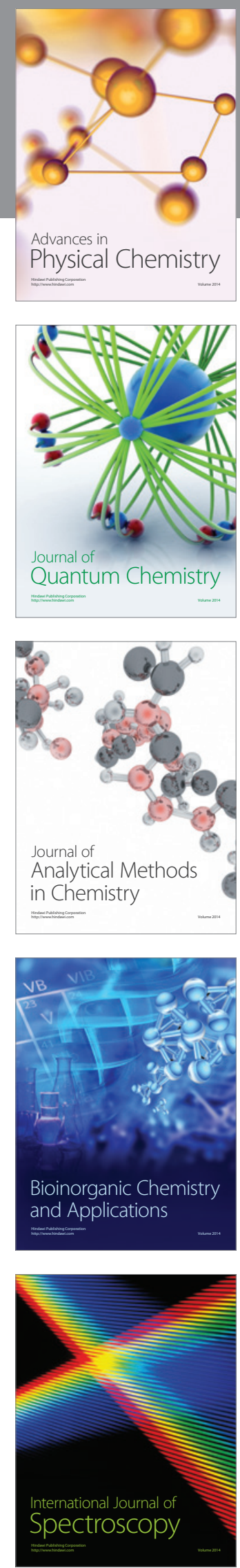